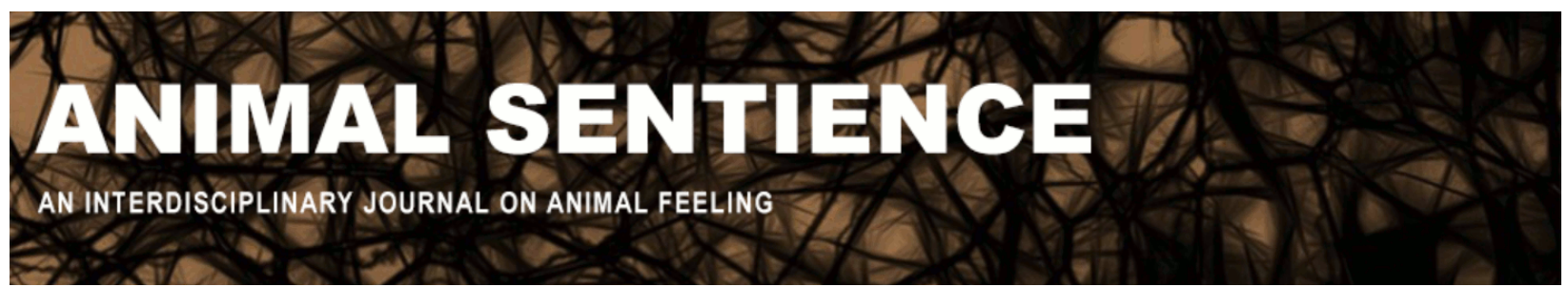

Merker, Bjorn H. (2016) Insects join the consciousness fray. Animal Sentience 9(4)

DOI: $10.51291 / 2377-7478.1131$

Date of submission: 2016-08-03

Date of acceptance: 2016-08-05

(c)

This article has appeared in the journal Animal

Sentience, a peer-reviewed journal on animal

cognition and feeling. It has been made open access,

free for all, by WellBeing International and deposited

in the WBI Studies Repository. For more information,

please contact

wbisr-info@wellbeingintl.org.

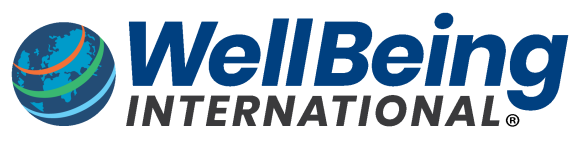

SOLUTIONS FOR PEOPLE, ANIMALS AND ENVIRONMENT 


\title{
Insects join the consciousness fray
}

Commentary on Klein \& Barron on Insect Experience

\author{
Bjorn Merker \\ Kristianstad, Sweden
}

\begin{abstract}
Klein \& Barron's review of recent insect neurobiology helps correct the impression that insect behavior is orchestrated without the benefit of central integrative mechanisms. Given their existence, the authors go on to ask whether these central mechanisms also feature the kind of integrative operations that support sentience, and propose that they do. Along the way they raise a number of conceptual and evidentiary issues of fundamental importance for the neuroscience of consciousness, allowing me to comment favorably on a number of them. I conclude by pointing to ways in which the conception of insect sentience they outline might be tested empirically.
\end{abstract}

\begin{abstract}
Bjorn Merker is a neuroscientist with longstanding interest in brain mechanisms of consciousness: He has worked on subcortical mechanisms of orienting behavior in rodents and cats, mirror self-recognition in gibbons, and structural principles intrinsic to the neural organization of a conscious state. Fjälkestadsv. 410-82, SE-29194 Kristianstad, Sweden. https://en.wikipedia.org/wiki/Björn_Merker

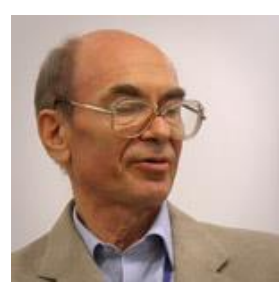

Progress in mapping the phylogenetic distribution of sentience has been hampered by a paucity of evidence about central integrative neural arrangements in species other than vertebrates. It has even been claimed that members of a major taxonomic group of invertebrates, the insects, function without central integrative mechanisms of the kind typical of vertebrates (Altman \& Kien, 1989; Merker, 2007; but see Merker, 2005, p. 107). To the extent that conceptions of sentience rely on central integrative functions, this would imply that such animals might be insentient altogether.

Not so, according to Klein \& Barron (2016) and the earlier work of theirs on which they build (Barron \& Klein, 2016). By reviewing recent evidence for integrative functional arrangements in the insect brain, they open avenues for exploring the bearing of that evidence on the question of insect sentience. Their adoption, in so doing, of a minimal definition of sentience as the capacity for mere experience is well taken, and is - as they themselves point out more than an expedient tailored to limitations of "lowly" insects. It is, rather, a matter of principle, because the issue of what kinds of neural arrangements are needed to implement a sentient state of any kind is prior to all questions regarding what contents might occupy such a state. It is, in other words, foundational for the neuroscience of consciousness.

One aspect of this foundational issue concerns whether neuron numbers are critical for the neural implementation of sentience. The authors are to be commended for discounting the 
significance of neuron numbers in this regard. If a sentience state is a function of particular structural arrangements of neurons, those arrangements might be implemented on vastly different scales, with neuron number serving variables such as the sophistication and resolution of the contents of that state, or the learning-based nature of those contents. An instructive analogy for a function reliant on a scaleable structural arrangement is DNA replication. As a function of the double-stranded structure of DNA, held together by weak hydrogen bonds, the mechanism of replication works the same way whether short sequences of few base pairs or long ones composed of millions are being replicated. Similarly, if sentience is a function of particular structural arrangements of neural operations, these may be present in brains of vastly different size.

There is also no reason to believe that the mechanism of sentience even in large brains requires more than a subset, even a small subset, of available neurons. The entire content of a monocular, monochromatic human visual percept at full psychophysical resolution is a matter of only some 164,000 pixels when the fall-off in acuity with eccentricity is taken into account (Rojer \& Schwartz, 1990; see also Merker, 2013, p. 3). In principle it could therefore be implemented by the same number of neurons serving as picture elements. There is, moreover, no reason to believe that a visual percept needs to have anything close to our level of resolution to be useful.

Why such a percept needs to be experienced in order to be useful is of course the central crux of consciousness theory. One answer to that question is that mobile, spatially orienting animals stand to reap energetic savings by integrating target selection, action selection, and motivational ranking at a central convergent interface for behavioral decision-making. Functional considerations favor a doubly nested arrangement of that interface, such that a decision-making egocenter is lodged within a neural body map, which is in turn lodged within a neural world map. When held together by a shared egocentric coordinate system such an arrangement yields the first-person perspective of confronting a world from inside a body, which is the typical condition of sentience (Merker, 2007, 2013. For circumstances under which the body-map requirement may be relaxed, see Merker, 2005, p. 107).

Klein \& Barron's treatment goes a long way towards making midline-straddling portions of the insect protocerebrum plausible candidates for the implementation of such a first-person interface. It is now possible to explore this proposal along both physiological and behavioral lines. Physiological studies are needed to determine whether the critical informational modalities that converge in this part of the insect brain indeed do so by interfacing in a shared egocentric coordinate space, so that their joint operation yields a sentient state. And behavioral studies are needed to delineate the role played by this candidate mechanism in the functional economy of insect behavior as a whole. To this end several types of behavior reviewed by Brembs (2011) as evidence for operationally well-defined "free will" in insects might be explored.

Brembs himself downplays the potential bearing of these behaviors on the issue of sentience. It is worth noting, however, that they typically occur in the waking state only. Given the possibility of insect sentience, an insect analog of "absence epilepsy" would partition this waking state into episodes supporting only automatisms on the one hand (insentient wakefulness), and episodes featuring the behaviors addressed by Brembs on the 
other (sentient wakefulness). This would be as close to a demonstration of sentience in insects as a third-person perspective is likely to take us. Klein \& Barron are to be congratulated on opening the door to such tantalizing vistas.

\section{References}

Altman, J. S., \& Kien, J. (1989). New models for motor control. Neural Computation, 1, 173183.

Barron, A. B., \& Klein, C. (2016). What insects can tell us about the origins of consciousness. Proceedings of the National Academy of Science of the United States of America, 113(18), 4900-4908.

Brembs, B. (2011). Towards a scientific concept of free will as a biological trait: Spontaneous actions and decision-making in invertebrates. Proceedings of the Royal Society London (B), 278, 930-939. doi:10.1098/rspb.2010.2325

Klein, C., \& Barron, A. B. (2016). Insects have the capacity for subjective experience. Animal Sentience 2016.100.

Merker, B. (2005). The liabilities of mobility: A selection pressure for the transition to consciousness in animal evolution. Consciousness and Cognition, 14, 89-114.

Merker, B. (2007). Consciousness without a cerebral cortex: A challenge for neuroscience and medicine. Target article, commentaries and author's response. The Behavioral and Brain Sciences, 30, 63-134.

Merker, B. (2013). The efference cascade, consciousness, and its self: Naturalizing the first person pivot of action control. Frontiers in Psychology 4, article 501, 1-20.

Rojer, A. S., \& Schwartz, E. L. (1990). Design characteristics for a space-variant visual sensor with complex-logarithmic geometry. In Proceedings 10th International Conference on Pattern Recognition (1990), Vol.2 (Los Alamitos, CA: IEEE Computer Society Press), 278285. 